TRANSACTIONS OF THE

AMERICAN MATHEMATICAL SOCIETY

Volume 363, Number 7, July 2011, Pages 3601-3620

S 0002-9947(2011)05221-9

Article electronically published on February 1, 2011

\title{
NODAL SOLUTIONS FOR SOME SINGULARLY PERTURBED DIRICHLET PROBLEMS
}

\author{
TERESA D'APRILE AND ANGELA PISTOIA
}

\begin{abstract}
We consider the equation $-\varepsilon^{2} \Delta u+u=f(u)$ in a bounded, smooth domain $\Omega \subset \mathbb{R}^{N}$ with homogeneous Dirichlet boundary conditions. We prove the existence of nodal solutions with multiple peaks concentrating at different points of $\Omega$. The nonlinearity $f$ grows superlinearly and subcritically. We do not require symmetry conditions on the geometry of the domain.
\end{abstract}

\section{INTRODUCTION}

Let us consider the following singularly perturbed elliptic problem:

$$
\left\{\begin{array}{lr}
\varepsilon^{2} \Delta u-u+|u|^{p-2} u=0 & \text { in } \Omega, \\
u=0 & \text { on } \partial \Omega,
\end{array}\right.
$$

where $\Omega$ is a smooth bounded domain of $\mathbb{R}^{N}, N \geq 2,2<p<\frac{2 N}{N-2}$ if $N \geq 3$ and $p>2$ if $N=2$, and $\varepsilon>0$ is a small parameter. Problem (1.1) arises in various mathematical models deriving from biological population theory, chemical reactor theory, etc. There have been many results which prove that solutions of (1.1) may exhibit sharp peaks near a certain number of points. In particular many papers have been devoted to establishing the existence of single and multiple peak solutions and to determining the location of the peaks as well as the profile of the spikes as $\varepsilon \rightarrow 0^{+}$. Ni and Wei in 23 showed that for $\varepsilon>0$ sufficiently small problem (1.1) has a positive least energy solution $v_{\varepsilon}$ which develops a spike layer at the most centered part of the domain, i.e. $\mathrm{d}_{\partial \Omega}\left(P_{\varepsilon}\right) \rightarrow \max _{P \in \Omega} \mathrm{d}_{\partial \Omega}(P)$, denoting by $P_{\varepsilon}$ the unique maximum of $v_{\varepsilon}$. Hereafter $\mathrm{d}_{\partial \Omega}(P)$ denotes the distance of $P$ from $\partial \Omega$. Since then, there have been many papers looking for higher energy solutions. More specifically, several papers study the effect of the geometry of the domain on the existence of positive solutions with single and multiple peaks (see [3, 4, 7], [8], 9], 10], 11, 14, 15], 17], 21], 26] and the references therein). However, in a general domain the existence of $k$-peaked positive solutions when $k \geq 2$ is not guaranteed. Indeed, in [10] the authors showed that if $\Omega$ is a strictly convex domain, problem (1.1) does not admit $k$-peaked positive solutions for any $k \geq 2$ (see also [25] when $k=2$ ).

Received by the editors December 16, 2008 and, in revised form, October 1, 2009.

2010 Mathematics Subject Classification. Primary 35B40, 35J20, 35J57.

Key words and phrases. Nodal solutions, multiple peaks, finite-dimensional reduction.

The authors were supported by Mi.U.R. project "Metodi variazionali e topologici nello studio di fenomeni non lineari". 
We point out that, for the corresponding Neumann problem, multiple interior and boundary positive spikes in a general domain have been constructed, for example, in [5], [18, [19], 20], and 22].

On the other hand, multiple peak nodal solutions always exist for problem (1.1) in a bounded and smooth domain $\Omega$. The first result was due to Noussair and Wei in 24, where it is proved that for $\varepsilon$ sufficiently small (1.1) has a least energy nodal solution with one positive boundary peak $P_{1}^{\varepsilon}$ and one negative boundary peak $P_{2}^{\varepsilon}$ whose location depends on the geometry of the $\Omega$. More precisely, if $\bar{P}_{1}:=\lim _{\varepsilon \rightarrow 0} P_{1}^{\varepsilon}$ and $\bar{P}_{2}:=\lim _{\varepsilon \rightarrow 0} P_{1}^{\varepsilon}$, then $\varphi\left(\bar{P}_{1}, \bar{P}_{2}\right)=\max _{\left(P_{1}, P_{2}\right) \in \Omega \times \Omega} \varphi\left(P_{1}, P_{2}\right)$, where the function $\varphi: \Omega \times \Omega \stackrel{\varepsilon \rightarrow 0}{\rightarrow} \mathbb{R}$ is defined by

$$
\varphi\left(P_{1}, P_{2}\right):=\min \left\{\mathrm{d}_{\partial \Omega}\left(P_{1}\right), \mathrm{d}_{\partial \Omega}\left(P_{2}\right), \frac{\left|P_{1}-P_{2}\right|}{2}\right\} .
$$

In 27] the authors showed that in the case of the unit ball such a solution is odd in one direction. We also mention the papers [1]-2] where, by a different approach, a lower bound on the number of sign-changing solutions is provided; however, these papers are not concerned with the shape of such solutions. As far as we know the question of the existence of $k$-peaked nodal solutions for problem (1.1) with $k \geq 3$, without symmetry assumptions on the domain $\Omega$, is largely open. It is the purpose of the present paper to establish this kind of result.

We are able to deal with more general nonlinearities. We consider the problem

$$
\left\{\begin{array}{lr}
-\varepsilon^{2} \Delta u+u=f(u) & \text { in } \Omega, \\
u=0 & \text { on } \partial \Omega .
\end{array}\right.
$$

We will assume that $f: \mathbb{R} \rightarrow \mathbb{R}$ is of class $\mathcal{C}^{1+\sigma}$ and satisfies the following conditions:

(f1) $f(0)=f^{\prime}(0)=0$ and $f(u)=-f(-u)$ for any $u \in \mathbb{R}$.

(f2) $f(u)=O\left(|u|^{p_{1}}\right), f^{\prime}(u)=O\left(|u|^{p_{2}-1}\right)$ as $|u| \rightarrow \infty$ for some $p_{1}, p_{2}>1$ and there exists $p_{3}>1$ such that

$$
\left|f^{\prime}(u+\phi)-f^{\prime}(u)\right| \leq \begin{cases}c|\phi|^{p_{3}-1} & \text { if } p_{3}>2, \\ c\left(|\phi|+|\phi|^{p_{3}-1}\right) & \text { if } p_{3} \leq 2 .\end{cases}
$$

(f3) The problem

$$
\left\{\begin{array}{l}
\Delta w-w+f(w)=0, \quad w>0 \text { in } \mathbb{R}^{N} \\
w(0)=\max _{z \in \mathbb{R}^{N}} w(z), \quad \lim _{|z| \rightarrow+\infty} w(z)=0
\end{array}\right.
$$

has a unique solution $w$, which is nondegenerate; i.e., denoting by $L$ the linearized operator

$$
L: H^{2}\left(\mathbb{R}^{N}\right) \rightarrow L^{2}\left(\mathbb{R}^{N}\right), \quad L[u]:=\Delta u-u+f^{\prime}(w) u,
$$

then

$$
\operatorname{Kernel}(L)=\operatorname{span}\left\{\frac{\partial w}{\partial z_{1}}, \ldots, \frac{\partial w}{\partial z_{N}}\right\} .
$$

By the well-known result of Gidas, Ni and Nirenberg $([16]), w$ is radially symmetric and strictly decreasing in $r=|z|$. Moreover, by classical regularity results, the 
following asymptotic result holds:

$$
\lim _{|z| \rightarrow+\infty}|z|^{\frac{N-1}{2}} e^{|z|} w(|z|)=A>0 \text { and } \lim _{|z| \rightarrow+\infty} \frac{w^{\prime}(|z|)}{w(|z|)}=-1 .
$$

The main result of this paper states that, given two positive integers $k_{1}, k_{2}$ with $k_{1}+k_{2} \leq 6$ (with the exception of the couples $(1,5)$ and $(5,1)$ in the two-dimensional case), for $\varepsilon$ sufficiently small (1.2) possesses a solution with $k_{1}$ positive peaks and $k_{2}$ negative peaks approaching different points of $\Omega$. Furthermore, each peak has a profile similar to $w$ suitably rescaled. More precisely we will prove the following theorem.

Theorem 1.1. Assume that hypotheses (f1)-(f3) hold and that $\Omega \subset \mathbb{R}^{N}(N \geq 2)$ is a smooth and bounded domain. Let $k_{1}, k_{2} \in \mathbb{N}$ satisfy

$$
k_{1}, k_{2} \geq 1, \quad k:=k_{1}+k_{2} \leq 6, \quad\left(k_{1}, k_{2}\right) \neq(1,5),(5,1) \text { if } N=2 .
$$

Then, for $\varepsilon>0$ sufficiently small, the problem (1.2) has a solution $v_{\varepsilon} \in H^{2}(\Omega)$.

Furthermore, there exist $P_{1}^{\varepsilon}, \ldots, P_{k}^{\varepsilon} \in \Omega$ such that, as $\varepsilon \rightarrow 0^{+}$,

(a) $v_{\varepsilon}(x)=\sum_{i=1}^{k_{1}} w\left(\frac{x-P_{i}^{\varepsilon}}{\varepsilon}\right)-\sum_{i=k_{1}+1}^{k} w\left(\frac{x-P_{i}^{\varepsilon}}{\varepsilon}\right)+o\left(e^{-\frac{\delta}{\varepsilon}}\right)$ uniformly for $x \in \bar{\Omega}$,

(b) $\left|P_{i}^{\varepsilon}-P_{h}^{\varepsilon}\right| \geq \delta$ for $i \neq h, \mathrm{~d}_{\partial \Omega}\left(P_{i}^{\varepsilon}\right) \geq \delta$ for all $i$

for a suitable $\delta>0$.

An easy computation shows that we obtain 9 pairs of nodal peak solutions for problem (1.2) if $N \geq 3$ and 8 pairs if $N=2$, provided that $\varepsilon$ is small enough.

We would like to remark that in the presence of symmetries we can localize the limit concentration points $\bar{P}_{i}:=\lim _{\varepsilon \rightarrow 0} P_{i}^{\varepsilon}, i=1, \ldots, k$, as it is proved in Section 5 , However, in general we can only conjecture that the location of the peaks is strictly related with the location of suitable critical points of the function $\varphi: \Omega^{k} \rightarrow \mathbb{R}$ defined by

$$
\varphi\left(P_{1}, \ldots, P_{k}\right):=\min _{\substack{i, h=1, \ldots, k \\ i \neq h}}\left\{\mathrm{~d}_{\partial \Omega}\left(P_{i}\right), \frac{\left|P_{i}-P_{h}\right|}{2}\right\} .
$$

More precisely, we conjecture that $\left(\bar{P}_{1}, \ldots, \bar{P}_{k}\right)$ is a critical point of $\varphi$ such that

$$
\frac{\left|\bar{P}_{i}-\bar{P}_{h}\right|}{2}>\varphi\left(\bar{P}_{1}, \ldots, \bar{P}_{k}\right) \quad \text { if } \quad \text { either } i, h=1, \ldots, k_{1} \quad \text { or } \quad i, h=k_{1}+1, \ldots, k \text {. }
$$

Finally, we point out that a multiplicity result for nodal multi-peak solutions has been obtained in 12 for the Dirichlet problem in the presence of a positive potential $V$ and in [13] for the corresponding Neumann problem. In both cases the peaks collapse to the same point, contrary to the present situation.

To introduce the main idea of the proof of Theorem 1.1 we first need to fix some notation. For any smooth bounded domain $U$, let $\mathcal{P}_{U} w$ be the projection of $w$ into $\mathrm{H}_{0}^{1}(U)$, i.e.

$$
\left\{\begin{array}{lr}
-\Delta u+u=f(w) & \text { in } U \\
u=0 & \text { on } \partial U .
\end{array}\right.
$$

For $P \in \Omega$ we set

$$
\Omega_{\varepsilon}:=\{y: \varepsilon y \in \Omega\} \text { and } \Omega_{\varepsilon, P}:=\{y: \varepsilon y+P \in \Omega\} .
$$


Let us scale problem (1.2), so that we get an equivalent problem

$$
\left\{\begin{array}{lr}
-\Delta u+u=f(u) & \text { in } \Omega_{\varepsilon}, \\
u=0 & \text { on } \partial \Omega_{\varepsilon} .
\end{array}\right.
$$

Associated with problem (1.8) is the rescaled energy functional

$$
J_{\varepsilon}(u):=\frac{1}{2} \int_{\Omega_{\varepsilon}}\left(|\nabla u|^{2}+u^{2}\right)-\int_{\Omega_{\varepsilon}} F(u), \quad u \in \mathrm{H}_{0}^{1}\left(\Omega_{\varepsilon}\right),
$$

where $F(u)=\int_{0}^{u} f(s) d s$. Fix $\mathbf{P}=\left(P_{1}, \ldots, P_{k}\right) \in \Omega^{k}$ and set, for any $i=1, \ldots, k$,

$$
w_{i}(y)=w_{P_{i}}(y):=w\left(y-\frac{P_{i}}{\varepsilon}\right) \text { and } \mathcal{P} w_{i}(y):=\mathcal{P}_{\Omega_{\varepsilon}} w_{i}(y), y \in \Omega_{\varepsilon} .
$$

We look for a solution to (1.8) as

$$
u=\sum_{i=1}^{k} \lambda_{i} \mathcal{P} w_{i}+\Phi_{\varepsilon, \mathbf{P}}
$$

where $\lambda_{i} \in\{-1,+1\}$ and the rest term $\Phi_{\varepsilon, \mathbf{P}}=\Phi_{\varepsilon, P_{1}, \ldots, P_{k}}$ belongs to a suitable space. By using the classical Lyapunov-Schmidt reduction method, in order to find such a solution the location of the concentration points $P_{1}, \ldots, P_{k}$ should be critical for the reduced functional

$\tilde{J}_{\varepsilon}(\mathbf{P})=k I(w)+\frac{1}{2}(\gamma+o(1)) \sum_{i=1}^{k} e^{-2 \frac{\mathrm{d} \partial \Omega\left(P_{i}\right)}{\varepsilon}}-\frac{1}{2}(\gamma+o(1)) \sum_{i \neq h} \lambda_{i} \lambda_{h} w\left(\frac{P_{i}-P_{h}}{\varepsilon}\right)+$ h.o.t.

on a suitable open set $\mathcal{D}_{\varepsilon} \subset \Omega^{k}$, where $\gamma$ is a positive constant. The terms $e^{-2 \frac{\mathrm{d} \partial \Omega}{\varepsilon}\left(P_{i}\right)}$ represent the boundary effect on each peak, created by the boundary condition, while the terms $-\lambda_{i} \lambda_{h} w\left(\frac{P_{i}-P_{h}}{\varepsilon}\right)$ are due to the interaction among the peaks which has an attractive or a repulsive effect according to its respective sign.

Let us observe that the sum $\sum_{i=1}^{k} e^{-2 \frac{\mathrm{d} \partial \Omega\left(P_{i}\right)}{\varepsilon}}$ tends to cluster the points $P_{i}$ at the most centered part of $\Omega$, while, using (1.5), the sum $\sum_{\lambda_{i}=-\lambda_{h}} w\left(\frac{P_{i}-P_{h}}{\varepsilon}\right)$ tends to repel the points $P_{i}$ from each other. Therefore, in the case $k_{1}=k_{2}=1$ we have $\lambda_{i} \lambda_{h}=\lambda_{1} \lambda_{2}=-1$, and then we can easily conclude that the two sums above achieve an equilibrium for a suitable configuration of the points $P_{1}, P_{2}$, which is a local minimum for the functional $\tilde{J}_{\varepsilon}$.

In the general case $k_{1}, k_{2} \geq 1$ the presence of the term $-\sum_{\lambda_{i}=\lambda_{h}} w\left(\frac{P_{i}-P_{h}}{\varepsilon}\right)$ does not allow us to catch a critical point of $\tilde{J}_{\varepsilon}$ as a local minimum or a local maximum. However the different interaction effects of the boundary and the peaks provide $\tilde{J}_{\varepsilon}$ with a suitable local linking structure. Following the strategy of [12], we will use a max-min theorem to obtain the existence of a saddle point for $\tilde{J}_{\varepsilon}$. The choice of the set where we minimize $\tilde{J}_{\varepsilon}$ will be crucial. Roughly speaking, such a set is made up of configurations $P_{1}, \ldots, P_{k}$ for which the terms $w\left(\frac{P_{i}-P_{h}}{\varepsilon}\right)$ with $\lambda_{i}=\lambda_{h}$ $(i \neq h)$ are negligible (for example, in the case $k_{1}=k_{2}$, we choose the set of the configurations which are aligned on a fixed direction with alternating sign), so that the remaining terms may balance.

Once we have obtained a local geometrical linking structure, in order to apply a max-min argument to conclude the existence of a critical point a kind of compactness property for $\tilde{J}_{\varepsilon}$ is required. More precisely, we need to prove that the 
tangential component of the gradient of $\tilde{J}_{\varepsilon}$ on $\partial \mathcal{D}_{\varepsilon}$ is not zero at the max-min level. This is the most technical part of the paper, and the choice of $\mathcal{D}_{\varepsilon}$ plays a key role in our computations. A restriction on the number of peaks $k_{1}+k_{2} \leq 6$, which occurs in Theorem 1.1, is required in this last step. Indeed, for larger numbers the method breaks down due to lack of compactness arising in some configurations, which are related to the solutions of certain sphere-packing type variational problem (see Proposition 4.1). Such crucial configurations may appear, for example, when 7 of the $k$ points $\left(P_{1}, \ldots, P_{k}\right)$ are placed in a hexagonal arrangement (i.e. a single point surrounded by 6 other points at the vertices of a regular hexagon). The difficulty due to this loss of compactness for $k_{1}+k_{2} \geq 7$ seems the main obstacle in producing solutions to the equation (1.1) with a higher number of mixed positive and negative peaks when no symmetry on the domain $\Omega$ is assumed.

Since the Lyapunov-Schmidt procedure has been widely used, in this paper we shall only sketch the process, referring to [5] and [19] for the details, and concentrate on the max-min part.

The paper is organized as follows. Notation, preliminaries and some useful estimates are recalled in Section 2. In Section 3 we sketch the Lyapunov-Schmidt reduction method which reduces the problem to a finite-dimensional problem. Section 4 is devoted to applying a max-min argument to the reduced functional $\tilde{J}_{\varepsilon}$ in order to catch a critical point. Finally, in Section 5, we deal with the symmetric case, where the use of natural constraints allows us to improve the above results for general domains.

\section{Preliminaries}

We need the following results (see 23, Lemma 4.4). Set

$\varphi_{\varepsilon, P}(x):=w\left(\frac{x-P}{\varepsilon}\right)-\mathcal{P}_{\Omega_{\varepsilon, P}} w\left(\frac{x-P}{\varepsilon}\right)$ and $\psi_{\varepsilon, P}(x):=-\varepsilon \log \varphi_{\varepsilon, P}(x), x \in \Omega$.

\section{Lemma 2.1.}

$$
\psi_{\varepsilon, P}(x) \rightarrow \psi_{0}(x):=\inf _{z \in \partial \Omega}\{|z-P|+L(x, z)\} \text { as } \varepsilon \rightarrow 0
$$

uniformly in $\bar{\Omega}$, where $L(x, z)$ is the infimum of $T$ such that there exists $\xi(s) \in$ $\mathcal{C}^{0,1}([0, T], \bar{\Omega})$ with $\xi(0)=x, \xi(T)=z,\left|\frac{d \xi}{d s}\right| \leq 1$ a.e. in $[0, T]$. In particular $\psi_{0}(P)=2 \mathrm{~d}_{\partial \Omega}(P)$.

Let

$$
V_{\varepsilon, P}(y):=\frac{\varphi_{\varepsilon, P}(\varepsilon y+P)}{\varphi_{\varepsilon, P}(P)}, y \in \Omega_{\varepsilon, P}
$$

Lemma 2.2. For every sequence $\varepsilon_{n} \rightarrow 0$, there is a subsequence $\varepsilon_{n h} \rightarrow 0$ such that $V_{\varepsilon_{n h}, P} \rightarrow \widetilde{V}$ uniformly on every compact set of $\mathbb{R}^{N}$, where $\widetilde{V}$ is a positive solution of

Furthermore, for any $\sigma_{1}>0$,

$$
\begin{cases}\Delta u-u=0 & \text { in } \mathbb{R}^{N} \\ u>0 & \text { in } \mathbb{R}^{N} \\ u(0)=1 & \end{cases}
$$

$$
\sup _{y \in \Omega_{\varepsilon_{n h}, P}} e^{-\left(1+\sigma_{1}\right)|y|}\left|V_{\varepsilon, P}(y)-\widetilde{V}(y)\right| \rightarrow 0 \quad \text { as } \quad \varepsilon_{n h} \rightarrow 0 .
$$


Moreover, the following result holds (see [26], Lemma 2.4).

Lemma 2.3. Assume that $P_{\varepsilon} \in \Omega$ is such that $P_{\varepsilon} \rightarrow P$ as $\varepsilon \rightarrow 0$ and $P \in \Omega$. Then there exists a bounded Borel measure $d \mu_{P}$ on $\partial \Omega$ with

$$
\int_{\partial \Omega} d \mu_{P}=1 \quad \text { and } \operatorname{supp}\left(d \mu_{P}\right) \subset \Pi_{\partial \Omega}(P):=\left\{Q \in \partial \Omega:|Q-P|=\mathrm{d}_{\partial \Omega}(P)\right\}
$$

such that, up to a subsequence,

$$
V_{\varepsilon, P_{\varepsilon}}(y) \rightarrow \widetilde{V}(y):=\int_{\partial \Omega} e^{\left\langle\frac{Q-P}{|Q-P|}, y\right\rangle} d \mu_{P} \text { as } \varepsilon \rightarrow 0 .
$$

It is well known that the distance function $\mathrm{d}_{\partial \Omega}: \Omega \rightarrow \mathbb{R}$ is a Lipschitz continuous function, and by [6], Theorem 2.8.2, we deduce that for any $P \in \Omega$

$$
\begin{gathered}
\partial \mathrm{d}_{\partial \Omega}(P)=\left\{\int_{\partial \Omega} \nu(Q) d \mu_{P}(Q): d \mu_{P} \text { is a bounded Borel measure on } \partial \Omega,\right. \\
\left.\int_{\partial \Omega} d \mu_{P}(Q)=1 \text { and } \operatorname{supp}\left(d \mu_{P}\right) \subset \Pi_{\partial \Omega}(P)\right\},
\end{gathered}
$$

where $\Pi_{\partial \Omega}(P):=\left\{Q \in \partial \Omega:|Q-P|=\mathrm{d}_{\partial \Omega}(P)\right\}$ and $\nu(Q)$ denotes the unit inward normal at the point $Q \in \partial \Omega$.

\section{The Lyapunov-Schmidt Reduction}

Let $k \geq 1$ be a fixed integer. Let $\Lambda_{\delta}:=\left\{\mathbf{P}:=\left(P_{1}, \ldots, P_{k}\right): \varphi(\mathbf{P}) \geq \delta\right\}$ for some small $\delta>0$, where the function $\varphi$ is defined in (1.6).

Fix $\mathbf{P} \in \Lambda_{\delta}$ and set

$$
\begin{aligned}
& \mathcal{K}_{\varepsilon, \mathbf{P}}=\operatorname{span}\left\{\frac{\partial \mathcal{P} w_{i}}{\partial P_{i}^{j}}: i=1, \ldots, k, j=1, \ldots, N\right\} \subset \mathrm{H}^{2}\left(\Omega_{\varepsilon}\right) \cap \mathrm{H}_{0}^{1}\left(\Omega_{\varepsilon}\right), \\
& \mathcal{C}_{\varepsilon, \mathbf{P}}=\operatorname{span}\left\{\frac{\partial \mathcal{P} w_{i}}{\partial P_{i}^{j}}: i=1, \ldots, k, j=1, \ldots, N\right\} \subset \mathrm{L}^{2}\left(\Omega_{\varepsilon}\right),
\end{aligned}
$$

denoting by $P_{i}^{j}$ the $j$-th component of $P_{i}$ for $j=1, \ldots, N$. We also need the following spaces:

$$
\begin{aligned}
& \mathcal{K}_{\varepsilon, \mathbf{P}}^{\perp}=\left\{u \in \mathrm{H}^{2}\left(\Omega_{\varepsilon}\right) \cap \mathrm{H}_{0}^{1}\left(\Omega_{\varepsilon}\right): \int_{\Omega_{\varepsilon}} u \frac{\partial \mathcal{P} w_{i}}{\partial P_{i}^{j}}=0, i=1, \ldots, k, j=1, \ldots, N\right\}, \\
& \mathcal{C}_{\varepsilon, \mathbf{P}}^{\perp}=\left\{u \in \mathrm{L}^{2}\left(\Omega_{\varepsilon}\right): \int_{\Omega_{\varepsilon}} u \frac{\partial \mathcal{P} w_{i}}{\partial P_{i}^{j}}=0, i=1, \ldots, k, j=1, \ldots, N\right\} .
\end{aligned}
$$

First of all, we point out that solving problem (1.8) is equivalent to solving

$$
S_{\varepsilon}(u):=\Delta u+u-f(u)=0, \quad u \in \mathrm{H}^{2}\left(\Omega_{\varepsilon}\right) \cap \mathrm{H}_{0}^{1}\left(\Omega_{\varepsilon}\right) .
$$

We have the following result. 
Proposition 3.1. For any $\delta>0$ there exist $\varepsilon_{0}>0$ and $c>0$ such that for any $\varepsilon \in\left(0, \varepsilon_{0}\right)$ and for any $\boldsymbol{P} \in \Lambda_{\delta}$ there exists a unique $\Phi_{\varepsilon, \boldsymbol{P}} \in \mathcal{K}_{\varepsilon, \boldsymbol{P}}^{\perp}$ such that

$$
S_{\varepsilon}\left(\sum_{i=1}^{k} \lambda_{i} \mathcal{P} w_{i}+\Phi_{\varepsilon, \boldsymbol{P}}\right) \in \mathcal{C}_{\varepsilon, \boldsymbol{P}}^{\perp}
$$

Moreover,

$$
\left\|\Phi_{\varepsilon, P}\right\|_{\mathrm{H}^{2}\left(\Omega_{\varepsilon}\right)} \leq c \varepsilon^{-\left(1+\frac{\sigma}{2}\right) \frac{\varphi(P)}{\varepsilon}} .
$$

Finally, the function $\boldsymbol{P} \rightarrow \Phi_{\varepsilon, \boldsymbol{P}} \in \mathrm{H}_{0}^{1}\left(\Omega_{\varepsilon}\right)$ is of class $\mathcal{C}^{1}$.

Proof. We argue exactly as in [19, Section 3, and [5], Section 3.

Let us introduce the reduced energy $\widetilde{J}_{\varepsilon}: \Lambda_{\delta} \rightarrow \mathbb{R}$ as

$$
\widetilde{J}_{\varepsilon}(\mathbf{P}):=J_{\varepsilon}\left(\sum_{i=1}^{k} \lambda_{i} \mathcal{P} w_{i}+\Phi_{\varepsilon, \mathbf{P}}\right) .
$$

The following result holds.

Proposition 3.2. If $\boldsymbol{P} \in \Lambda_{\delta}$ is a critical point of $\widetilde{J}_{\mathcal{\varepsilon}}$, then the function $u_{\varepsilon}=$ $\sum_{i=1}^{k} \lambda_{i} \mathcal{P} w_{i}+\Phi_{\varepsilon, \boldsymbol{P}}$ is a critical point of $J_{\varepsilon}$, i.e. a solution to problem (1.8).

Proof. We argue exactly as in [5], Proposition 3.6.

We need the expansion of the reduced energy.

Lemma 3.3. It holds that

$$
\widetilde{J}_{\varepsilon}(\boldsymbol{P})=k I(w)+\frac{1}{2}(\gamma+o(1)) \sum_{i=1}^{k} \varphi_{\varepsilon, P_{i}}\left(P_{i}\right)-\frac{1}{2}(\gamma+o(1)) \sum_{\substack{i, h=1 \\ i \neq h}}^{k} \lambda_{i} \lambda_{h} w\left(\frac{\left|P_{i}-P_{h}\right|}{\varepsilon}\right)
$$

uniformly with respect to $\boldsymbol{P} \in \Lambda_{\delta}$. Here

$$
\begin{aligned}
& I(w):=\frac{1}{2} \int_{\mathbb{R}^{N}}\left(|\nabla w|^{2}+w^{2}\right) d y-\int_{\mathbb{R}^{N}} F(w) d y, \\
& \gamma:=\frac{1}{2} \int_{\mathbb{R}^{N}} f(w) e^{-y_{1}} d y .
\end{aligned}
$$

Proof. We argue exactly as in Lemma 2.6 and Proposition 4.1 of [19].

Lemma 3.4. If $h=1, \ldots, k$ and $j=1, \ldots, N$, it holds that

$$
\begin{aligned}
\frac{\partial \widetilde{J}_{\varepsilon}}{\partial P_{h}^{j}}(\boldsymbol{P})= & -\frac{\gamma}{\varepsilon}\left[\alpha\left(P_{h}\right)\right]^{j} \varphi_{\varepsilon, P_{h}}\left(P_{h}\right)+\frac{\gamma}{\varepsilon} \sum_{\substack{i=1 \\
i \neq h}}^{k} \lambda_{i} \lambda_{h} \frac{P_{h}^{j}-P_{i}^{j}}{\left|P_{h}-P_{i}\right|} w\left(\frac{\left|P_{i}-P_{h}\right|}{\varepsilon}\right) \\
& +\frac{1}{\varepsilon} o\left(\sum_{i=1}^{k} \varphi_{\varepsilon, P_{i}}\left(P_{i}\right)+\sum_{\substack{i, l=1 \\
i \neq l}}^{k} w\left(\frac{\left|P_{i}-P_{l}\right|}{\varepsilon}\right)\right)
\end{aligned}
$$


uniformly with respect to $\boldsymbol{P} \in \Lambda_{\delta}$, where $\alpha\left(P_{h}\right) \in \partial \mathrm{d}_{\partial \Omega}\left(P_{h}\right)$ (see (2.1)) and $\left[\alpha\left(P_{h}\right)\right]^{j}$ denotes the $j$-th component of $\alpha\left(P_{h}\right)$.

Proof. Arguing exactly as in Lemma 4.2 of [5], if $h=1, \ldots, k$ and $j=1, \ldots, N$, we have

$$
\begin{aligned}
\frac{\partial \widetilde{J}_{\varepsilon}}{\partial P_{h}^{j}}(\mathbf{P})= & J_{\varepsilon}^{\prime}\left(\sum_{i=1}^{k} \lambda_{i} \mathcal{P} w_{i}+\Phi_{\varepsilon, \mathbf{P}}\right)\left[\lambda_{h} \frac{\partial \mathcal{P} w_{h}}{\partial P_{h}^{j}}+\frac{\partial \Phi_{\varepsilon, \mathbf{P}}}{\partial P_{h}^{j}}\right] \\
= & J_{\varepsilon}^{\prime}\left(\sum_{i=1}^{k} \lambda_{i} \mathcal{P} w_{i}\right)\left[\lambda_{h} \frac{\partial \mathcal{P} w_{h}}{\partial P_{h}^{j}}\right] \\
& +\left[J_{\varepsilon}^{\prime}\left(\sum_{i=1}^{k} \lambda_{i} \mathcal{P} w_{i}+\Phi_{\varepsilon, \mathbf{P}}\right)-J_{\varepsilon}^{\prime}\left(\sum_{i=1}^{k} \lambda_{i} \mathcal{P} w_{i}\right)\right]\left[\lambda_{h} \frac{\partial \mathcal{P} w_{h}}{\partial P_{h}^{j}}\right] \\
& +J_{\varepsilon}^{\prime}\left(\sum_{i=1}^{k} \lambda_{i} \mathcal{P} w_{i}+\Phi_{\varepsilon, \mathbf{P}}\right)\left[\frac{\partial \Phi_{\varepsilon, \mathbf{P}}}{\partial P_{h}^{j}}\right] \\
= & J_{\varepsilon}^{\prime}\left(\sum_{i=1}^{k} \lambda_{i} \mathcal{P} w_{i}\right)\left[\lambda_{h} \frac{\partial \mathcal{P} w_{h}}{\partial P_{h}^{j}}\right]+\text { h.o.t. }
\end{aligned}
$$

where

$$
\text { h.o.t }=\frac{1}{\varepsilon} o\left(\sum_{i=1}^{k} \varphi_{\varepsilon, P_{i}}\left(P_{i}\right)+\sum_{\substack{i, l=1 \\ i \neq l}}^{k} w\left(\frac{\left|P_{i}-P_{l}\right|}{\varepsilon}\right)\right) .
$$

It remains to estimate the leading term of (3.5):

$$
\begin{aligned}
& J_{\varepsilon}^{\prime}\left(\sum_{i=1}^{k} \lambda_{i} \mathcal{P} w_{i}\right)\left[\lambda_{h} \frac{\partial \mathcal{P} w_{h}}{\partial P_{h}^{j}}\right] \\
& =\sum_{i=1}^{k} \lambda_{i} \lambda_{h} \int_{\Omega_{\varepsilon}}\left(\nabla \mathcal{P} w_{i} \nabla \frac{\partial \mathcal{P} w_{h}}{\partial P_{h}^{j}}+\mathcal{P} w_{i} \frac{\partial \mathcal{P} w_{h}}{\partial P_{h}^{j}}\right) d y-\int_{\Omega_{\varepsilon}} f\left(\sum_{i=1}^{k} \lambda_{i} \mathcal{P} w_{i}\right) \lambda_{h} \frac{\partial \mathcal{P} w_{h}}{\partial P_{h}^{j}} \\
& =\sum_{i=1}^{k} \lambda_{i} \lambda_{h} \int_{\Omega_{\varepsilon}} f\left(w_{i}\right) \frac{\partial \mathcal{P} w_{h}}{\partial P_{h}^{j}}-\int_{\Omega_{\varepsilon}} f\left(\sum_{i=1}^{k} \lambda_{i} \mathcal{P} w_{i}\right) \lambda_{h} \frac{\partial \mathcal{P} w_{h}}{\partial P_{h}^{j}} \\
& =\sum_{\substack{i=1 \\
i \neq h}}^{k} \lambda_{i} \lambda_{h} \int_{\Omega_{\varepsilon}} f\left(w_{i}\right) \frac{\partial \mathcal{P} w_{h}}{\partial P_{h}^{j}}+\int_{\Omega_{\varepsilon}}\left[f\left(w_{h}\right)-f\left(\mathcal{P} w_{h}\right)\right] \frac{\partial \mathcal{P} w_{h}}{\partial P_{h}^{j}} \\
& \quad-\int_{\Omega_{\varepsilon}}\left[f\left(\sum_{i=1}^{k} \lambda_{i} \mathcal{P} w_{i}\right)-f\left(\lambda_{h} \mathcal{P} w_{h}\right)\right] \lambda_{h} \frac{\partial \mathcal{P} w_{h}}{\partial P_{h}^{j}}
\end{aligned}
$$

Arguing as in Lemma 4.1 of [17] (see estimate (4.6)) we get

$$
\int_{\Omega_{\varepsilon}}\left[f\left(w_{h}\right)-f\left(\mathcal{P} w_{h}\right)\right] \frac{\partial \mathcal{P} w_{h}}{\partial P_{h}^{j}}=-\gamma\left[\alpha\left(P_{h}\right)\right]^{j} \frac{\varphi_{\varepsilon, P_{h}}\left(P_{h}\right)}{\varepsilon}+o\left(\frac{\varphi_{\varepsilon, P_{h}}\left(P_{h}\right)}{\varepsilon}\right),
$$


where $\gamma$ is defined in (3.3) and $\alpha\left(P_{h}\right):=\int_{\partial \Omega} \frac{Q-P}{|Q-P|} d \mu_{P} \in \partial \mathrm{d}_{\partial \Omega}\left(P_{h}\right)$ (because of Lemma 2.3 and (2.1)).

Arguing as in Lemma 4.1 of [5] (see the proof of estimate (4.2)) we get for $i \neq h$

$$
\int_{\Omega_{\varepsilon}} f\left(w_{i}\right) \frac{\partial \mathcal{P} w_{h}}{\partial P_{h}^{j}}=\frac{\gamma}{\varepsilon} w\left(\frac{\left|P_{i}-P_{h}\right|}{\varepsilon}\right) \frac{P_{i}-P_{h}}{\left|P_{i}-P_{h}\right|}+\text { h.o.t. }
$$

and arguing as in Lemma 4.2 of [5] (see the estimate of $I_{3}$ ) we get

$$
\begin{aligned}
& \int_{\Omega_{\varepsilon}}\left[f\left(\sum_{i=1}^{k} \lambda_{i} \mathcal{P} w_{i}\right)-f\left(\lambda_{h} \mathcal{P} w_{h}\right)\right] \lambda_{h} \frac{\partial \mathcal{P} w_{h}}{\partial P_{h}^{j}} \\
& =2 \frac{\gamma}{\varepsilon} \sum_{\substack{i=1 \\
i \neq h}}^{k} \lambda_{i} \lambda_{h} w\left(\frac{\left|P_{i}-P_{h}\right|}{\varepsilon}\right) \frac{P_{i}-P_{h}}{\left|P_{i}-P_{h}\right|}+\text { h.o.t. }
\end{aligned}
$$

Collecting all the previous estimates, the claim follows.

By Lemma 3.3 and Lemma 3.4 we deduce the following remark.

Remark 3.5. For any $i=1, \ldots, k$ and $j=1, \ldots, N$ it holds that

$$
J_{\varepsilon}\left(\mathcal{P} w_{i}\right)=I(w)+\frac{1}{2}(\gamma+o(1)) \varphi_{\varepsilon, P_{i}}\left(P_{i}\right)
$$

and

$$
\frac{\partial}{\partial P_{i}^{j}} J_{\varepsilon}\left(\mathcal{P} w_{i}\right)=-\frac{\gamma}{\varepsilon}\left[\alpha\left(P_{i}\right)\right]^{j} \varphi_{\varepsilon, P_{i}}\left(P_{i}\right)(1+o(1))
$$

uniformly with respect to $P_{i}$ in compact sets of $\Omega$, where $\alpha\left(P_{i}\right) \in \partial \mathrm{d}_{\partial \Omega}\left(P_{i}\right)$ (see (2.1) ).

\section{The MAX-Min ARgument}

According to Proposition 3.2 we just need to prove that the reduced functional $\tilde{J}_{\varepsilon}$ has a critical point to find a solution of (1.2). Let $k_{1}, k_{2} \geq 1$ be such that

$$
k:=k_{1}+k_{2} \leq 6 \quad \text { and } \quad\left(k_{1}, k_{2}\right) \neq(1,5),(5,1) \text { if } N=2 .
$$

We need the following auxiliary lemma.

Lemma 4.1. There exists a continuous function

$$
\begin{aligned}
& \mathbf{S}: \mathbb{R}^{N} \times(0, \infty)^{k-1} \longrightarrow \mathbb{R}^{k N}, \\
& (P, \mathbf{r}) \longmapsto \mathbf{S}_{P, \mathbf{r}}=\left(S_{1}(P, \mathbf{r}), \ldots, S_{k}(P, \mathbf{r})\right)
\end{aligned}
$$

such that, setting $\mathbf{r}=\left(r_{2}, \ldots, r_{k}\right)$,

$$
\begin{gathered}
r_{h}=\min _{i<h}\left|S_{h}(P, \mathbf{r})-S_{i}(P, \mathbf{r})\right|, \quad h=2, \ldots, k, \\
\left|S_{i}(P, \mathbf{r})-S_{h}(P, \mathbf{r})\right| \geq \sqrt{2} \min _{i=2, \ldots, k} r_{i} \quad \text { if } \lambda_{i} \lambda_{h}=1 \text { and } i \neq h, \\
\left|S_{i}(P, \mathbf{r})-P\right| \leq k \max _{2 \leq i \leq k} r_{i} .
\end{gathered}
$$


Proof. For the sake of definiteness, we will assume $k_{1} \leq k_{2}$ and set

$$
\lambda_{i}=(-1)^{i+1} \text { if } i=1, \ldots, 2 k_{1}, \quad \lambda_{i}=-1 \text { if } i=2 k_{1}+1, \ldots, k .
$$

If $\left\{\mathbf{e}_{1}, \ldots, \mathbf{e}_{N}\right\}$ denote the standard basis in $\mathbb{R}^{N}$, let us choose $k_{2}-k_{1}+1$ vectors (note that $k_{2}-k_{1}+1 \leq 2 N$ by (4.10) $Z_{1}, Z_{2 k_{1}+1}, \ldots, Z_{k}$ such that

$$
\left\{Z_{1}, Z_{2 k_{1}+1}, \ldots, Z_{k}\right\} \subset\left\{ \pm \mathbf{e}_{1}, \pm \mathbf{e}_{2}, \ldots, \pm \mathbf{e}_{N}\right\}, \quad Z_{i} \neq Z_{h} \text { for } i \neq h .
$$

We define $\mathbf{S}$ in the following way: for any $P \in \mathbb{R}^{N}$ and $\mathbf{r}=\left(r_{2}, \ldots, r_{k}\right) \in(0, \infty)^{k-1}$ set

$$
\begin{cases}S_{1}(P, \mathbf{r})=P, & \\ S_{i}(P, \mathbf{r})=P+\sum_{s=2}^{i} r_{s} Z_{1} & \text { if } 2 \leq i \leq 2 k_{1}, \\ S_{i}(P, \mathbf{r})=P+r_{i} Z_{i} & \text { if } 2 k_{1}+1 \leq i \leq k .\end{cases}
$$

We remark that it holds that

$$
\left|S_{h}(P, \mathbf{r})-S_{i}(P, \mathbf{r})\right|^{2} \begin{cases}=\left(r_{i+1}+\cdots+r_{h}\right)^{2} & \text { if } 1 \leq i<h \leq 2 k_{1}, \\ \geq\left(r_{2}+\cdots+r_{i}\right)^{2}+r_{h}^{2} & \text { if } 2 \leq i \leq 2 k_{1}<h \leq k \\ \geq r_{i}^{2}+r_{h}^{2} & \text { if } 2 k_{1}+1 \leq i<h \leq k\end{cases}
$$

and

$$
\left|S_{i}(P, \mathbf{r})-P\right|= \begin{cases}0 & \text { if } i=1 \\ r_{2}+\cdots+r_{i} & \text { if } 2 \leq i \leq 2 k_{1} \\ r_{i} & \text { if } 2 k_{1}+1 \leq i \leq k\end{cases}
$$

By (4.6) and (4.7), recalling (4.5), we deduce (4.2)-(4.4).

We want to apply a max-min argument to characterize a topologically nontrivial critical value of $\tilde{J}_{\varepsilon}$. More precisely, we are going to prove the existence of sets $\mathcal{D}_{\varepsilon}$, $K, K_{0} \subset \mathbb{R}^{k N}$ satisfying the following properties:

$(\mathrm{P} 1) \mathcal{D}_{\varepsilon}$ is an open set with smooth boundary $\partial \mathcal{D}_{\varepsilon}, K_{0}$ and $K$ are compact sets, $K$ is connected and

$$
K_{0} \subset K \subset \mathcal{D}_{\varepsilon} \subset \overline{\mathcal{D}}_{\varepsilon} \subset \Omega^{k} .
$$

(P2) If we define the complete metric space $\mathcal{F}$ by

$$
\mathcal{F}=\left\{\eta: K \rightarrow \mathcal{D}_{\varepsilon} \mid \eta \text { continuous, } \eta(\mathbf{P})=\mathbf{P} \forall \mathbf{P} \in K_{0}\right\},
$$

then

$$
c_{\varepsilon}:=\sup _{\eta \in \mathcal{F}} \min _{\mathbf{P} \in K} \tilde{J}_{\varepsilon}[\eta(\mathbf{P})]<\min _{\mathbf{P} \in K_{0}} \tilde{J}_{\varepsilon}[\mathbf{P}] .
$$

(P3) For every $\mathbf{P} \in \partial \mathcal{D}_{\varepsilon}$ such that $\tilde{J}_{\varepsilon}[\mathbf{P}]=c_{\varepsilon}$, there exists a vector $\tau_{\mathbf{P}}$ tangent to $\partial \mathcal{D}_{\varepsilon}$ at $\mathbf{P}$ so that $\partial_{\tau_{\mathbf{P}}} \tilde{J}_{\varepsilon}[\mathbf{P}] \neq 0$.

Under these assumptions a critical point $\mathbf{P}_{\varepsilon} \in \mathcal{D}_{\varepsilon}$ of $\tilde{J}_{\varepsilon}$ with $\tilde{J}_{\varepsilon}\left[\mathbf{P}_{\varepsilon}\right]=c_{\varepsilon}$ exists, as a standard deformation argument involving the gradient flow of $\tilde{J}_{\varepsilon}$ shows. 
4.1. Definition of the sets $\mathcal{D}_{\varepsilon}, K, K_{0}$. Let $\delta>0$ be a sufficiently small number and define 1

$\mathcal{D}_{\varepsilon}=\left\{\mathbf{P} \in \Omega^{k} \mid F_{\varepsilon}(\mathbf{P}):=\sum_{i=1}^{k} J_{\varepsilon}\left(\mathcal{P} w_{i}\right)+\frac{\gamma}{2} \sum_{\substack{i, h=1 \\ i \neq h}}^{k} w\left(\frac{\left|P_{i}-P_{h}\right|}{\varepsilon}\right)<k I(w)+e^{-\frac{3 \delta}{\varepsilon}}\right\}$

$$
=\left\{\mathbf{P} \in \Omega^{k} \mid \frac{1}{2}(\gamma+o(1)) \sum_{i=1}^{k} e^{-\frac{\psi_{\varepsilon}\left(P_{i}\right)}{\varepsilon}}+\frac{\gamma}{2} \sum_{\substack{i, h=1 \\ i \neq h}}^{k} w\left(\frac{\left|P_{i}-P_{h}\right|}{\varepsilon}\right)<e^{-\frac{3 \delta}{\varepsilon}}\right\},
$$

because of Remark 3.5 .

Observe that by Lemma 2.1 and (1.5)

$$
\mathcal{D}_{\varepsilon} \subset\left\{\mathbf{P} \in \Omega^{k}|| P_{i}-P_{h} \mid \geq 2 \delta \forall i \neq h, \quad \mathrm{~d}_{\partial \Omega}\left(P_{i}\right) \geq \delta\right\} .
$$

Moreover, we easily deduce that

$$
-\varepsilon \log \left(\sum_{i=1}^{k} e^{-\frac{\psi_{\varepsilon}\left(P_{i}\right)}{\varepsilon}}+\sum_{\substack{i, h=1 \\ i \neq h}}^{k} w\left(\frac{\left|P_{i}-P_{h}\right|}{\varepsilon}\right)\right) \rightarrow 2 \varphi(\mathbf{P}) \text { as } \varepsilon \rightarrow 0,
$$

where $\varphi$ is defined in (1.6).

Then let $W_{\varepsilon}$ be the following open set of $\mathbb{R}^{N+k-1}$ :

$$
W_{\varepsilon}=\left\{(P, \mathbf{r}) \mid \mathbf{S}_{P, \mathbf{r}} \in \Omega^{k}, F_{\varepsilon}\left(\mathbf{S}_{P, \mathbf{r}}\right)<k I(w)+\frac{1}{2} e^{-\frac{3 \delta}{\varepsilon}}\right\},
$$

where $\mathbf{S}_{P, \mathbf{r}}$ is defined in Lemma 4.1

Let $P_{0} \in \Omega$ be such that $\mathrm{d}_{\partial \Omega}\left(P_{0}\right)=\max _{P \in \Omega} \mathrm{d}_{\partial \Omega}\left(P_{0}\right)$. Let us point that

$$
\left(P_{0}, \mathbf{r}_{0}\right) \in W_{\varepsilon} \text {, where } \mathbf{r}_{0}:=\left(r_{0}, \ldots, r_{0}\right) \in \mathbb{R}^{k-1} \text { and } r_{0}=\frac{\mathrm{d}_{\partial \Omega}\left(P_{0}\right)}{k+1} \text {. }
$$

Indeed, according to (4.2), $\left|S_{i}\left(P_{0}, \mathbf{r}_{0}\right)-S_{h}\left(P_{0}, \mathbf{r}_{0}\right)\right| \geq \frac{\mathrm{d} \Omega \Omega\left(P_{0}\right)}{k+1}$ for $i \neq h$, and by (1.5) we immediately check $w\left(\frac{\left|S_{i}\left(P_{0}, \mathbf{r}_{0}\right)-S_{h}\left(P_{0}, \mathbf{r}_{0}\right)\right|}{\varepsilon}\right)=o\left(e^{-\frac{\mathrm{d} \partial \Omega\left(P_{0}\right)}{\varepsilon(k+1)}}\right)$ for $i \neq h$. Moreover, according to (4.4) $\left|S_{i}\left(P_{0}, \mathbf{r}_{0}\right)-P_{0}\right| \leq \frac{k \mathrm{~d}_{\partial \Omega}\left(P_{0}\right)}{k+1}$, which implies $\mathrm{d}_{\partial \Omega}\left(S_{i}\left(P_{0}, \mathbf{r}_{0}\right)\right) \geq$ $\frac{\mathrm{d}_{\partial \Omega}\left(P_{0}\right)}{k+1}$, and consequently, by Lemma 2.1 , we deduce $\varphi_{\varepsilon, S_{i}\left(P_{0}, \mathbf{r}_{0}\right)}\left(S_{i}\left(P_{0}, \mathbf{r}_{0}\right)\right)=(1+$ $o(1)) e^{-\frac{\mathrm{d} \partial \Omega\left(S_{i}\left(P_{0}, \mathbf{r}_{0}\right)\right)}{\varepsilon}}=O\left(e^{-\frac{2 \mathrm{~d} \partial \Omega\left(P_{0}\right)}{\varepsilon(k+1)}}\right)$. Hence Remark 3.5 gives $J_{\varepsilon}\left(\mathcal{P} w_{S_{i}(P, \mathbf{r})}\right)=$ $I(w)+O\left(e^{-\frac{\mathrm{d} \partial \Omega}{\varepsilon(k+1)}}\right)$. If we choose $3 \delta<\frac{\mathrm{d}_{\partial \Omega}\left(P_{0}\right)}{k+1}$, we obtain $\left(P_{0}, \mathbf{r}_{0}\right) \in W_{\varepsilon}$.

Let $U_{\varepsilon}$ be the connected component of $W_{\varepsilon}$ containing $\left(P_{0}, \mathbf{r}_{0}\right)$ and let us define

$$
K=\left\{\mathbf{S}_{P, \mathbf{r}} \in \mathbb{R}^{k N} \mid(P, \mathbf{r}) \in \bar{U}_{\varepsilon}\right\} \text { and } K_{0}=\left\{\mathbf{S}_{P, \mathbf{r}} \in \mathbb{R}^{k N} \mid(P, \mathbf{r}) \in \partial U_{\varepsilon}\right\} .
$$

$K$ is connected and closed by construction since $U_{\varepsilon}$ is connected and closed. Furthermore, it is obvious that $K_{0} \subset K \subset \mathcal{D}_{\varepsilon} . K_{0}$ can be rewritten as

$$
K_{0}=\left\{\mathbf{S}_{P, \mathbf{r}} \mid(P, \mathbf{r}) \in \bar{U}_{\varepsilon}, \quad F_{\varepsilon}\left(\mathbf{S}_{P, \mathbf{r}}\right)=k I(w)+\frac{1}{2} e^{-\frac{3 \delta}{\varepsilon}}\right\} .
$$

\footnotetext{
${ }^{1}$ Hereafter, for the sake of simplicity, we will write $\psi_{\varepsilon}(P)$ in place of $\psi_{\varepsilon, P}(P)$.
} 
It is useful to point out that

$$
\tilde{J}_{\varepsilon}\left[\mathbf{S}_{P, \mathbf{r}}\right] \geq k I(w)+\gamma(1+o(1)) \sum_{\substack{i, h=1 \\ i \neq h}}^{k} w\left(\frac{\left|S_{i}(P, \mathbf{r})-S_{h}(P, \mathbf{r})\right|}{\varepsilon}\right) \quad \text { unif. for } \mathbf{S}_{P, \mathbf{r}} \in K
$$

and

$$
\tilde{J}_{\varepsilon}\left[\mathbf{S}_{P, \mathbf{r}}\right]=F_{\varepsilon}\left[\mathbf{S}_{P, \mathbf{r}}\right]+o\left(e^{-\frac{3 \delta}{\varepsilon}}\right) \text { unif. for } \mathbf{S}_{P, \mathbf{r}} \in K .
$$

In fact, by (4.2) and (4.3) we get that there exist $\bar{\imath}, \bar{h}$ such that $\lambda_{\bar{\imath}}=-\lambda_{\bar{h}}, \mid S_{\bar{\imath}}(P, \mathbf{r})-$ $S_{\bar{h}}(P, \mathbf{r})\left|=\min _{i \neq h}\right| S_{i}(P, \mathbf{r})-S_{h}(P, \mathbf{r}) \mid$ and $\min _{i \neq h, \lambda_{i}=\lambda_{h}}\left|S_{i}(P, \mathbf{r})-S_{h}(P, \mathbf{r})\right|>$ $\sqrt{2}\left|S_{\bar{\imath}}(P, \mathbf{r})-S_{\bar{h}}(P, \mathbf{r})\right|$. Hence, by (1.5)

$$
\sum_{\substack{i, h=1 \\ i \neq h, \lambda_{i}=\lambda_{h}}}^{k} w\left(\frac{S_{i}(P, \mathbf{r})-S_{h}(P, \mathbf{r})}{\varepsilon}\right)=o\left(w\left(\frac{S_{\bar{l}}(P, \mathbf{r})-S_{\bar{h}}(P, \mathbf{r})}{\varepsilon}\right)\right),
$$

and, using Lemma 3.3 and Remark 3.5, (4.14)-4.15) follow.

4.2. Proof of (4.9). Let $\eta \in \mathcal{F}$; namely $\eta: K \rightarrow \mathcal{D}_{\varepsilon}$ is a continuous function such that $\eta(\mathbf{P})=\mathbf{P}$ for any $\mathbf{P} \in K_{0}$. Setting $\eta=\left(\eta_{1}, \ldots, \eta_{k}\right)$ where $\eta_{i}: K \rightarrow \mathbb{R}^{N}$, let $\tilde{\eta}: \bar{U}_{\varepsilon} \rightarrow \mathbb{R}^{N} \times \mathbb{R}^{k-1}$ be defined by

$$
\tilde{\eta}_{1}(P, \mathbf{r})=\eta_{1}\left(\mathbf{S}_{P, \mathbf{r}}\right) \quad \text { and } \quad \tilde{\eta}_{i}(P, \mathbf{r})=\min _{h<i}\left|\eta_{i}\left(\mathbf{S}_{P, \mathbf{r}}\right)-\eta_{h}\left(\mathbf{S}_{P, \mathbf{r}}\right)\right| \text { for } i=2, \ldots, k \text {. }
$$

First of all $\tilde{\eta}$ is a continuous function because of the continuity of $\eta$. Secondly, we claim that $\tilde{\eta}(P, \mathbf{r})=(P, \mathbf{r})$ for any $(P, \mathbf{r}) \in \partial U_{\varepsilon}$. In fact, if $(P, \mathbf{r}) \in \partial U_{\varepsilon}$, then by definition $\mathbf{S}_{P, \mathbf{r}} \in K_{0}$; consequently $\eta\left(\mathbf{S}_{P, \mathbf{r}}\right)=\mathbf{S}_{P, \mathbf{r}}$, by which

while, using (4.2), for $i \geq 2$

$$
\tilde{\eta}_{1}(P, \mathbf{r})=\eta_{1}\left(\mathbf{S}_{P, \mathbf{r}}\right)=S_{1}(P, \mathbf{r})=P,
$$

$$
\tilde{\eta}_{i}(P, \mathbf{r})=\min _{h<i}\left|\eta_{i}\left(\mathbf{S}_{P, \mathbf{r}}\right)-\eta_{h}\left(\mathbf{S}_{P, \mathbf{r}}\right)\right|=\min _{h<i}\left|S_{i}(P, \mathbf{r})-S_{h}(P, \mathbf{r})\right|=r_{i} .
$$

Hence the theory of the topological degree ensures that there exists $(\bar{P}, \overline{\mathbf{r}}) \in U_{\varepsilon}$ such that $\tilde{\eta}(\bar{P}, \overline{\mathbf{r}})=\left(P_{0}, \mathbf{r}_{0}\right)$; that is (see (4.12) $)$,

$$
\eta_{1}\left(\mathbf{S}_{\bar{P}, \overline{\mathbf{r}}}\right)=P_{0} \quad \text { and } \quad \min _{h<i}\left|\eta_{i}\left(\mathbf{S}_{\bar{P}, \overline{\mathbf{r}}}\right)-\eta_{h}\left(\mathbf{S}_{\bar{P}, \overline{\mathbf{r}}}\right)\right|=\frac{\mathrm{d}_{\partial \Omega}\left(P_{0}\right)}{k+1}, i=2, \ldots, k .
$$

In particular

$$
\left|\eta_{i}\left(\mathbf{S}_{\bar{P}, \overline{\mathbf{r}}}\right)-\eta_{h}\left(\mathbf{S}_{\bar{P}, \overline{\mathbf{r}}}\right)\right| \geq \frac{\mathrm{d}_{\partial \Omega}\left(P_{0}\right)}{k+1} \quad \text { if } \quad i \neq h,
$$

which implies (because of (1.5))

$$
w\left(\frac{\eta_{i}\left(\mathbf{S}_{\bar{P}, \overline{\mathbf{r}}}\right)-\eta_{h}\left(\mathbf{S}_{\bar{P}, \overline{\mathbf{r}}}\right)}{\varepsilon}\right)=o\left(e^{-\frac{\mathrm{d} \partial \Omega}{\varepsilon\left(P_{0}\right)}}\right) \quad \text { if } \quad i \neq h .
$$

Moreover, it is not difficult to check that

$$
\left|\eta_{i}\left(\mathbf{S}_{\bar{P}, \overline{\mathbf{r}}}\right)-P_{0}\right|=\left|\eta_{i}\left(\mathbf{S}_{\bar{P}, \overline{\mathbf{r}}}\right)-\eta_{1}\left(\mathbf{S}_{\bar{P}, \overline{\mathbf{r}}}\right)\right| \leq \frac{k}{k+1} \mathrm{~d}_{\partial \Omega}\left(P_{0}\right), \quad i=2, \ldots, k,
$$

by which $\mathrm{d}_{\partial \Omega}\left(\eta_{i}\left(\mathbf{S}_{\bar{P}, \overline{\mathbf{r}}}\right)\right) \geq \frac{\mathrm{d} \partial \Omega\left(P_{0}\right)}{k+1}$ and consequently, reasoning as above,

$$
J_{\varepsilon}\left(\mathcal{P} w_{\eta_{i}\left(\mathbf{S}_{\bar{P}, \overline{\mathbf{r}}}\right)}\right)=I(w)+O\left(e^{-\frac{\mathrm{d} \partial \Omega\left(P_{0}\right)}{\varepsilon(k+1)}}\right) .
$$


Then by Lemma 3.3, (4.16) and (4.17), we deduce that

$$
\min _{\mathbf{S}_{P, \mathbf{r}} \in K} \tilde{J}_{\varepsilon}\left[\eta\left(\mathbf{S}_{P, \mathbf{r}}\right)\right] \leq k I(w)+O\left(e^{-\frac{\mathrm{d} \partial \Omega}{\varepsilon\left(P_{0}\right)}}\right) .
$$

Hence

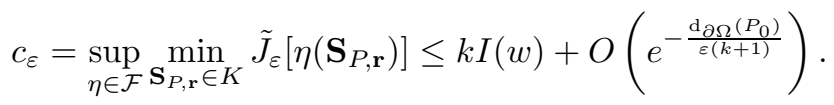

On the other hand, by taking $\eta\left(\mathbf{S}_{P, \mathbf{r}}\right)=\mathbf{S}_{P, \mathbf{r}}$ and using (4.14),

$$
c_{\varepsilon} \geq \min _{\mathbf{S}_{P, \mathbf{r}} \in K} \tilde{J}_{\varepsilon}\left[\mathbf{S}_{P, \mathbf{r}}\right] \geq k I(w) .
$$

Combining (4.18)-(4.19) we get

$$
k I(w) \leq c_{\varepsilon} \leq k I(w)+o\left(e^{-\frac{3 \delta}{\varepsilon}}\right) .
$$

On the other hand, combining (4.13) and (4.15)

$$
\min _{\mathbf{S}_{P, \mathbf{r}} \in K_{0}} \tilde{J}_{\varepsilon}\left[\mathbf{S}_{P, \mathbf{r}}\right]=k I(w)+\frac{1}{2}(1+o(1)) e^{-\frac{3 \delta}{\varepsilon}},
$$

and (4.9) follows.

4.3. Proof of (P3). Assume by contradiction that there exists $\mathbf{P} \in \partial \mathcal{D}_{\varepsilon}$ such that $\widetilde{J}_{\varepsilon}(\mathbf{P})=c_{\varepsilon}$ and $\tau_{\mathbf{P}} \cdot \nabla \widetilde{J}_{\varepsilon}(\mathbf{P})=0$ for any vector $\tau_{\mathbf{P}}$ tangent to $\partial \mathcal{D}_{\varepsilon}$ at $\mathbf{P}$. Therefore, there exists $\mu \in \mathbb{R}$ such that $\mathbf{P}$ is a critical point of the function

$$
\mathbf{P} \rightarrow \widetilde{J}_{\varepsilon}(\mathbf{P})+\mu \sum_{i=1}^{k} J_{\varepsilon}\left(\mathcal{P} w_{i}\right)+\mu \frac{\gamma}{2} \sum_{\substack{i, j=1 \\ i \neq j}}^{k} w\left(\frac{\left|P_{i}-P_{j}\right|}{\varepsilon}\right) .
$$

By Lemma 3.4. Remark 3.5 and estimate (1.5) we deduce that

$$
\begin{aligned}
& -\gamma \frac{\alpha\left(P_{h}\right)+\mu \beta\left(P_{h}\right)}{\varepsilon} e^{-\frac{\psi_{\varepsilon}\left(P_{h}\right)}{\varepsilon}}+\sum_{\substack{i=1 \\
i \neq h}}^{k} \gamma \frac{\lambda_{i} \lambda_{h}-\mu}{\varepsilon} \frac{P_{h}-P_{i}}{\left|P_{h}-P_{i}\right|} w\left(\frac{\left|P_{i}-P_{h}\right|}{\varepsilon}\right) \\
& \quad+o\left(\frac{e^{-\frac{3 \delta}{\varepsilon}}}{\varepsilon}\right)=0, h=1, \ldots, k,
\end{aligned}
$$

where $\alpha\left(P_{h}\right), \beta\left(P_{h}\right) \in \partial \mathrm{d}_{\partial \Omega}\left(P_{h}\right)$ (see (2.1)), which can be rewritten as

$$
\begin{aligned}
& -\left(\alpha\left(P_{h}\right)+\mu \beta\left(P_{h}\right)\right) e^{-\frac{\psi_{\varepsilon}\left(P_{h}\right)}{\varepsilon}}-(1+\mu) \sum_{\substack{i=1 \\
\lambda_{i} \lambda_{h}=-1}}^{k} \frac{P_{h}-P_{i}}{\left|P_{h}-P_{i}\right|} w\left(\frac{\left|P_{i}-P_{h}\right|}{\varepsilon}\right) \\
& +(1-\mu) \sum_{\substack{i=1 \\
i \neq h, \lambda_{i} \lambda_{h}=+1}}^{k} \frac{P_{h}-P_{i}}{\left|P_{h}-P_{i}\right|} w\left(\frac{\left|P_{i}-P_{h}\right|}{\varepsilon}\right)+o\left(e^{-\frac{3 \delta}{\varepsilon}}\right)=0, h=1, \ldots, k,
\end{aligned}
$$


or equivalently

$$
\begin{aligned}
& -\alpha\left(P_{h}\right) e^{-\frac{\psi_{\varepsilon}\left(P_{h}\right)}{\varepsilon}}-\sum_{\substack{i=1 \\
\lambda_{i} \lambda_{h}=-1}}^{k} \frac{P_{h}-P_{i}}{\left|P_{h}-P_{i}\right|} w\left(\frac{\left|P_{i}-P_{h}\right|}{\varepsilon}\right)+\sum_{\substack{i=1 \\
i \neq h, \lambda_{i} \lambda_{h}=1}}^{k} \frac{P_{h}-P_{i}}{\left|P_{h}-P_{i}\right|} w\left(\frac{\left|P_{i}-P_{h}\right|}{\varepsilon}\right) \\
& =\mu\left[\beta\left(P_{h}\right) e^{-\frac{\psi_{\varepsilon}\left(P_{h}\right)}{\varepsilon}}+\sum_{\substack{i=1 \\
i \neq h}}^{k} \frac{P_{h}-P_{i}}{\left|P_{h}-P_{i}\right|} w\left(\frac{\left|P_{i}-P_{h}\right|}{\varepsilon}\right)\right]+o\left(e^{-\frac{3 \delta}{\varepsilon}}\right), h=1, \ldots, k .
\end{aligned}
$$

Up to a subsequence, we can assume that $P_{i} \rightarrow \bar{P}_{i}$ as $\varepsilon \rightarrow 0$. Therefore

$$
\left|\bar{P}_{i}-\bar{P}_{j}\right| \geq 3 \delta \text { for } i \neq j \text { and } \mathrm{d}_{\partial \Omega}\left(\bar{P}_{i}\right) \geq \frac{3}{2} \delta .
$$

Let us point out that $\mu$ is bounded, and so (up to a subsequence) $\mu \rightarrow \bar{\mu}$ as $\varepsilon \rightarrow 0$. In fact, let $\mathbf{X}:=\left(X_{1}, \ldots, X_{k}\right)$, where

$$
\begin{aligned}
X_{h}:= & \beta\left(P_{h}\right) \frac{e^{-\frac{\psi_{\varepsilon}\left(P_{h}\right)}{\varepsilon}}}{\sum_{i=1}^{k} e^{-\frac{\psi_{\varepsilon}\left(P_{i}\right)}{\varepsilon}}+\sum_{\substack{i, j=1 \\
i \neq j}}^{k} w\left(\frac{\left|P_{i}-P_{j}\right|}{\varepsilon}\right)} \\
& +\sum_{\substack{i=1 \\
i \neq h}}^{k} \frac{P_{h}-P_{i}}{\left|P_{h}-P_{i}\right|} \frac{w\left(\frac{\left|P_{i}-P_{h}\right|}{\varepsilon}\right)}{\sum_{i=1}^{k} e^{-\frac{\psi_{\varepsilon}\left(P_{i}\right)}{\varepsilon}}+\sum_{\substack{i, j=1 \\
i \neq j}}^{k} w\left(\frac{\left|P_{i}-P_{j}\right|}{\varepsilon}\right)}, h=1, \ldots, k .
\end{aligned}
$$

By (4.22), taking into account (4.10), we obtain

$$
|\mu|\left|X_{h}\right|+o(1) \leq c,
$$

recalling $\left|\alpha\left(P_{h}\right)\right| \leq 1$ and $\mathbf{P} \in \partial \mathcal{D}_{\varepsilon}$. Moreover, since $\mathbf{P} \in \partial \mathcal{D}_{\varepsilon}$, by (4.11) we deduce that $\varphi(\overline{\mathbf{P}})=3 \delta / 2$ where $\overline{\mathbf{P}}:=\left(\bar{P}_{1}, \ldots, \bar{P}_{k}\right)$, and by Lemma 2.6 in [18 we deduce that $\mathbf{X} \rightarrow \overline{\mathbf{X}} \in \partial \varphi(\overline{\mathbf{P}})$. Finally, by Proposition 2.7 in $[18$ we get that if $\delta>0$ is small enough, then

$$
\inf \{|\mathbf{X}|: \mathbf{X} \in \partial \varphi(\mathbf{P}), \varphi(\mathbf{P}) \leq 3 \delta / 2\}>0 .
$$

(4.24) and (4.25) imply that $\mu$ is bounded.

Let

$$
a_{i h}:=\lim _{\varepsilon \rightarrow 0} w\left(\frac{\left|P_{i}-P_{h}\right|}{\varepsilon}\right) e^{\frac{3 \delta}{\varepsilon}} \text { and } c_{i}:=\lim _{\varepsilon \rightarrow 0} e^{-\frac{\psi_{\varepsilon}\left(P_{i}\right)}{\varepsilon}} e^{\frac{3 \delta}{\varepsilon}} .
$$

We point out that

$$
a_{i h}>0 \Rightarrow\left|\bar{P}_{i}-\bar{P}_{h}\right|=3 \delta
$$

and

$$
c_{i}>0 \Rightarrow 2 \mathrm{~d}_{\partial \Omega}\left(\bar{P}_{i}\right)=3 \delta \text { and } \partial \mathrm{d}_{\partial \Omega}\left(\bar{P}_{i}\right)=\left\{\nu_{i}\right\},
$$

where $\nu_{i}$ denotes the unit inward normal at the unique point $Q_{i} \in \partial \Omega$ such that $\left|Q_{i}-\bar{P}_{i}\right|=\mathrm{d}_{\partial \Omega}\left(\bar{P}_{i}\right)$. 
We multiply (4.21) by $e^{\frac{3 \delta}{\varepsilon}}$. Then we pass to the limit as $\varepsilon \rightarrow 0$ and get the system

$$
\begin{aligned}
& -\left(\alpha\left(\bar{P}_{h}\right)+\bar{\mu} \beta\left(\bar{P}_{h}\right)\right) c_{h}-(1+\bar{\mu}) \sum_{\substack{i=1 \\
\lambda_{i} \lambda_{h}=-1}}^{k} a_{i h} \frac{\bar{P}_{h}-\bar{P}_{i}}{\left|\bar{P}_{h}-\bar{P}_{i}\right|} \\
& +(1-\bar{\mu}) \sum_{\substack{i=1 \\
i \neq h, \lambda_{i} \lambda_{h}=+1}}^{k} a_{i h} \frac{\bar{P}_{h}-\bar{P}_{i}}{\left|\bar{P}_{h}-\bar{P}_{i}\right|}=0, h=1, \ldots, k .
\end{aligned}
$$

Here $\alpha\left(\bar{P}_{h}\right), \beta\left(\bar{P}_{h}\right) \in \partial \mathrm{d}_{\partial \Omega}\left(\bar{P}_{h}\right)$ (see (2.1)).

Let us point out that

$$
\sum_{\substack{i, j=1 \\ i \neq j, \lambda_{i} \lambda_{j}=+1}}^{k} w\left(\frac{\left|P_{i}-P_{j}\right|}{\varepsilon}\right) \geq C e^{-\frac{3 \delta}{\varepsilon}}
$$

for some constant $C>0$. In fact, by (4.20) we deduce that

$$
\sum_{i=1}^{k} e^{-\frac{\psi_{\varepsilon}\left(P_{i}\right)}{\varepsilon}}+\sum_{\substack{i, j=1 \\ \lambda_{i} \lambda_{j}=-1}}^{k} w\left(\frac{\left|P_{i}-P_{j}\right|}{\varepsilon}\right)-\sum_{\substack{i, j=1 \\ i \neq j, \lambda_{i} \lambda_{j}=+1}}^{k} w\left(\frac{\left|P_{i}-P_{j}\right|}{\varepsilon}\right)=o\left(e^{-\frac{3 \delta}{\varepsilon}}\right) .
$$

On the other hand, if $\mathbf{P} \in \partial \mathcal{D}_{\varepsilon}$, then

$(1+o(1)) \sum_{i=1}^{k} e^{-\frac{\psi_{\varepsilon}\left(P_{i}\right)}{\varepsilon}}+\sum_{\substack{i, j=1 \\ \lambda_{i} \lambda_{j}=-1}}^{k} w\left(\frac{\left|P_{i}-P_{j}\right|}{\varepsilon}\right)+\sum_{\substack{i, j=1 \\ i \neq j, \lambda_{i} \lambda_{j}=1}}^{k} w\left(\frac{\left|P_{i}-P_{j}\right|}{\varepsilon}\right)=\frac{2}{\gamma} e^{-\frac{3 \delta}{\varepsilon}}$.

If $\sum_{\substack{i, j=1 \\ i \neq j, \lambda_{i} \lambda_{j}=+1}}^{k} w\left(\frac{\left|P_{i}-P_{j}\right|}{\varepsilon}\right)=o\left(e^{-\frac{3 \delta}{\varepsilon}}\right)$, by (4.29) and (4.30) we will get a contradiction. By (4.28) we deduce that

$$
\sum_{\substack{i, j=1 \\ i \neq j, \lambda_{i} \lambda_{j}=+1}}^{k} a_{i j}>0
$$

Moreover, it holds that

$$
\sum_{i=1}^{k} e^{-\frac{\psi_{\varepsilon}\left(P_{i}\right)}{\varepsilon}}+\sum_{\substack{i, j=1 \\ \lambda_{i} \lambda_{j}=-1}}^{k} w\left(\frac{\left|P_{i}-P_{j}\right|}{\varepsilon}\right) \geq C e^{-\frac{3 \delta}{\varepsilon}}
$$

for some $C>0$. In fact, by (4.20) we deduce that $(4.33)$

$$
\sum_{i=1}^{k} e^{-\frac{\psi_{\varepsilon}\left(P_{i}\right)}{\varepsilon}}+\sum_{\substack{i, j=1 \\ \lambda_{i} \lambda_{j}=-1}}^{k} w\left(\frac{\left|P_{i}-P_{j}\right|}{\varepsilon}\right)-\sum_{\substack{i, j=1 \\ i \neq j, \lambda_{i} \lambda_{j}=1}}^{k} w\left(\frac{\left|P_{i}-P_{j}\right|}{\varepsilon}\right)+o\left(e^{-\frac{3 \delta}{\varepsilon}}\right) \geq 0
$$


If $\sum_{i=1}^{k} e^{-\frac{\psi_{\varepsilon}\left(P_{i}\right)}{\varepsilon}}+\sum_{\substack{i, j=1 \\ \lambda_{i} \lambda_{j}=-1}}^{k} w\left(\frac{\left|P_{i}-P_{j}\right|}{\varepsilon}\right)=o\left(e^{-\frac{3 \delta}{\varepsilon}}\right)$, by (4.28) and (4.33) we will get a contradiction. By (4.32) we achieve

$$
\sum_{i=1}^{k} c_{i}+\sum_{\substack{i, j=1 \\ i \neq j, \lambda_{i} \lambda_{j}=-1}}^{k} a_{i j}>0
$$

If $c_{i}=0$ for any $i=1, \ldots, k$, system (4.27) reduces to

$$
\sum_{\substack{i=1 \\ i \neq h}}^{k} A_{i h} \frac{\bar{P}_{h}-\bar{P}_{i}}{\left|\bar{P}_{i}-\bar{P}_{h}\right|}=0, \quad h=1, \ldots, k
$$

where

$$
A_{i h}:=\left\{\begin{aligned}
-(1+\bar{\mu}) a_{i h} & \text { if } \lambda_{i} \lambda_{h}=-1 \\
(1-\bar{\mu}) a_{i h} & \text { if } \lambda_{i} \lambda_{h}=+1 .
\end{aligned}\right.
$$

By (4.31) and (4.34) we deduce that $A_{i h} \neq 0$ for at least one pair of indexes $i$ and $h$. A contradiction arises because of Proposition 4.1 .

Assume now that $c_{i}>0$ for some $i$. Therefore system (4.27) reduces to the system

$$
\left\{\begin{array}{c}
-(1+\bar{\mu}) c_{h} \nu_{h}-(1+\bar{\mu}) \sum_{\substack{i=1 \\
\lambda_{i} \lambda_{h}=-1}}^{k} a_{i h} \frac{\bar{P}_{h}-\bar{P}_{i}}{\left|\bar{P}_{i}-\bar{P}_{h}\right|} \\
+(1-\bar{\mu}) \sum_{\substack{i=1 \\
i \neq h, \lambda_{i} \lambda_{h}=+1}}^{k} a_{i h} \frac{\bar{P}_{h}-\bar{P}_{i}}{\left|\bar{P}_{i}-\bar{P}_{h}\right|}=0, \text { if } c_{h}>0, \\
-(1+\bar{\mu}) \sum_{\substack{i=1 \\
\lambda_{i} \lambda_{h}=-1}}^{k} a_{i h} \frac{\bar{P}_{h}-\bar{P}_{i}}{\left|\bar{P}_{i}-\bar{P}_{h}\right|} \\
+(1-\bar{\mu}) \sum_{\substack{i=1 \\
i \neq h, \lambda_{i} \lambda_{h}=+1}}^{k} a_{i h} \frac{\bar{P}_{h}-\bar{P}_{i}}{\left|\bar{P}_{i}-\bar{P}_{h}\right|}=0, \text { otherwise. }
\end{array}\right.
$$

If $\bar{\mu}=-1$ we get the system $\sum_{\substack{i=1 \\ i \neq h, \lambda_{i} \lambda_{h}=+1}}^{k} a_{i h} \frac{\bar{P}_{h}-\bar{P}_{i}}{\left|P_{i}-P_{h}\right|}=0, h=1, \ldots, k$. By (4.31) we deduce that $a_{i h} \neq 0$ for at least a pair of indexes $i$ and $h$ with $\lambda_{i}=\lambda_{h}$, and a contradiction arises again because of Proposition 4.1 .

Assume $\bar{\mu} \neq-1$. Without loss of generality we can assume $c_{1}>0$. Let

$$
\mathcal{H}:=\{1\} \cup\left\{h \mid \exists i_{1}, \ldots, i_{l} \text { s.t. } a_{1 i_{1}}>0, a_{i_{1} i_{2}}>0, \ldots, a_{i_{l} h}>0\right\}
$$

and $\mathcal{I}:=\{1, \ldots, k\} \backslash \mathcal{H}$. $\mathcal{H}$ could reduce to $\{1\}$. It is easy to check that if $i \in \mathcal{I}$ and $h \in \mathcal{H}$, then $a_{i h}=0$. We point out that if $h \in \mathcal{H}$, then $\left|\bar{P}_{1}-\bar{P}_{h}\right| \leq 3 k \delta$ and 
$\mathrm{d}_{\partial \Omega}\left(\bar{P}_{h}\right) \leq(6 k+3) \delta / 2$. Therefore, if $\delta$ is small enough,

$$
\text { for any } h \in \mathcal{H} \quad \partial \mathrm{d}_{\partial \Omega}\left(\bar{P}_{h}\right)=\left\{\nu_{h}\right\} \text { and } \nu_{1} \cdot \nu_{h}>0 .
$$

Finally, by adding all the equations in system (4.36) with index $h \in \mathcal{H}$, we get $\sum_{h \in \mathcal{H}} c_{h} \nu_{h}=0$, and a contradiction arises because of (4.37). That concludes the proof.

Proposition 4.1. Let $k \geq 2, N \geq 2$ and consider the function

$$
\left(X_{1}, \ldots, X_{k}\right) \in \mathbb{R}^{N k} \longmapsto \sum_{\substack{i, h=1 \\ i \neq h}}^{k} A_{i h}\left|X_{i}-X_{h}\right|,
$$

where $A_{i h}=A_{h i}$. If (4.38) is not identically zero and there exists $\left(\bar{X}_{1}, \ldots, \bar{X}_{k}\right)$ a critical point of (4.38) satisfying

$$
\left|\bar{X}_{i}-\bar{X}_{h}\right| \geq 1 \text { for } i \neq h \quad \text { and } \quad\left|\bar{X}_{i}-\bar{X}_{h}\right|=1 \text { if } A_{i h} \neq 0,
$$

then $k \geq 7$.

Proof. See [12.

\section{The SYMMETRIC CASE}

Theorem 5.1. Assume that hypotheses (f1)-(f3) hold and that $\Omega$ is symmetric with respect to the reflection at $\mathbb{R} \times\{0\}$ and $\Omega \cap \mathbb{R} \times\{0\} \neq \emptyset$. Let $k \geq 1$ be a fixed integer. Then, for $\varepsilon>0$ sufficiently small, the problem (1.2) as a solution $v_{\varepsilon} \in H^{2}(\Omega)$.

Furthermore there exist $P_{i}^{\varepsilon} \in \Sigma:=\Omega \cap \mathbb{R} \times\{0\}, P_{i}^{\varepsilon}:=\left(t_{i}^{\varepsilon}, 0\right)$ with $t_{1}^{\varepsilon}<\cdots<t_{k}^{\varepsilon}$ such that, as $\varepsilon \rightarrow 0^{+}$,

(i) $v_{\varepsilon}(x)=\sum_{i=1}^{k}(-1)^{i} w\left(\frac{x-P_{i}^{\varepsilon}}{\varepsilon}\right)+o\left(e^{-\frac{\delta}{\varepsilon}}\right)$ uniformly for $x \in \bar{\Omega}$ for some $\delta>0$;

(ii) $\boldsymbol{t}^{\varepsilon}:=\left(t_{1}^{\varepsilon}, \ldots, t_{k}^{\varepsilon}\right) \rightarrow \boldsymbol{t}^{*}$ as $\varepsilon$ goes to zero, where

$$
\varphi\left(\boldsymbol{t}^{*}\right):=\max _{\substack{t \in \Sigma^{k} \\ t_{1}<\cdots<t_{k}}} \min _{i}\left\{\mathrm{~d}_{\partial \Sigma}\left(t_{i}\right), \frac{t_{i+1}-t_{i}}{2}\right\} .
$$

Proof. For any integer $k$ we look for a symmetric solution to (1.2) as in (1.10) with $\lambda_{i}=(-1)^{i}$ and

$$
P_{i}:=\left(t_{i}, 0\right), t_{i} \in \Sigma:=\Omega \cap \mathbb{R} \times\{0\} \quad \text { and } \quad t_{1}<\cdots<t_{k} .
$$

The rest term $\Phi_{\varepsilon, \mathbf{P}}$ will be chosen even with respect to the variables $x_{2}, \ldots, x_{N}$.

Arguing in a standard way, we are led to find a critical point of the reduced energy

$$
\widetilde{J}_{\varepsilon}(\mathbf{t})=k I(w)+\frac{1}{2}(\gamma+o(1)) \sum_{i=1}^{k} e^{-\frac{\psi_{\varepsilon}\left(t_{i}, 0\right)}{\varepsilon}}+(\gamma+o(1)) \sum_{i=2}^{k} w\left(\frac{t_{i}-t_{i-1}}{\varepsilon}\right),
$$

where the previous expansion holds uniformly on compact sets of $\left\{\mathbf{t} \in \Sigma^{k}: t_{1}<\right.$ $\left.\cdots<t_{k}\right\}$. Moreover, it is easy to check that $\widetilde{J}_{\varepsilon}$ has a minimum point $\mathbf{t}_{\varepsilon}$ on the set $\left\{\mathbf{t} \in \Sigma^{k}: t_{1}<\cdots<t_{k}\right\}$ and that (ii) holds.

Theorem 5.2. Assume that hypotheses (f1)-(f3) hold and that $\Omega$ is the unit ball. Let $5 \geq k \geq 1$ be a fixed integer. Then, for $\varepsilon>0$ sufficiently small, the problem (1.2) has a solution $v_{\varepsilon} \in H^{2}(\Omega)$. 
Furthermore, there exist $P_{j}^{\varepsilon}:=\left(\rho_{\varepsilon} e^{\frac{2 \pi \mathbf{i}}{k} j}, 0\right)$ (i denotes the imaginary unit), $j=$ $1, \ldots, k$, with $\rho_{\varepsilon} \in(0,1)$ such that, as $\varepsilon \rightarrow 0^{+}$,

(i) $v_{\varepsilon}(x)=\sum_{j=1}^{k} w\left(\frac{x-P_{j}^{\varepsilon}}{\varepsilon}\right)-w\left(\frac{x}{\varepsilon}\right)+o\left(e^{-\frac{\delta}{\varepsilon}}\right)$ uniformly for $x \in \bar{\Omega}$ for some $\delta>0$;

(ii) $\rho_{\varepsilon} \rightarrow \frac{2}{3}$ as $\varepsilon$ goes to zero.

Proof. For any integer $k$ we look for a symmetric solution to (1.2) as in (1.10) with $\lambda_{1}=\cdots=\lambda_{k}=+1$ and $\lambda_{k+1}=-1, P_{k+1}=0$ and $P_{j}=\left(\rho e^{\frac{2 \pi \mathbf{i}}{k} j}, 0\right)$, for $j=1, \ldots, k$ with $\rho \in(0,1)$. The rest term $\Phi_{\varepsilon, \mathbf{P}}$ is chosen even with respect to each $x_{h}, h=3, \ldots, N$, and, using the complex notation $z=x_{1}+\mathrm{i} x_{2}$,

$$
\Phi_{\varepsilon, \mathbf{P}}\left(z, x_{3}, \ldots, x_{N}\right)=\Phi_{\varepsilon, \mathbf{P}}\left(z e^{\frac{2 \pi \mathbf{i}}{k}}, x_{3}, \ldots, x_{N}\right) \quad \forall\left(z, x_{2}, \ldots x_{N}\right) \in \mathbb{R}^{N} .
$$

Arguing in a standard way, we are led to find a critical point of the reduced energy

$$
\begin{aligned}
\widetilde{J}_{\varepsilon}(\rho)= & k I(w)+\frac{1}{2}(\gamma+o(1)) e^{-\frac{\psi_{\varepsilon}(0)}{\varepsilon}}+\frac{k}{2}(\gamma+o(1)) e^{-\frac{\psi_{\varepsilon}\left(\rho e^{2 \pi \mathbf{i}, 0}\right)}{\varepsilon}} \\
& +k(\gamma+o(1)) w\left(\frac{\rho}{\varepsilon}\right)-k(\gamma+o(1)) \sum_{j=1}^{k-1} w\left(\rho \frac{\left|e^{2 \pi \mathbf{i}}-e^{\frac{2 \pi \mathbf{i}}{k} j}\right|}{\varepsilon}\right) \\
= & k I(w)+\frac{1}{2}(\gamma+o(1)) e^{-\frac{\psi_{\varepsilon}(0)}{\varepsilon}}+\frac{k}{2}(\gamma+o(1)) e^{-\frac{\psi_{\varepsilon}\left(\rho e^{2 \pi \mathbf{i}}, 0\right)}{\varepsilon}} \\
& +k(\gamma+o(1)) w\left(\frac{\rho}{\varepsilon}\right)-2 k(\gamma+o(1)) w\left(\rho \frac{\left(2-2 \cos \frac{2 \pi}{k}\right)^{1 / 2}}{\varepsilon}\right),
\end{aligned}
$$

where the previous expansion holds uniformly on compact sets of $(0,1)$. If $2-$ $2 \cos \frac{2 \pi}{k}>1$, i.e. $k<6$, by (5.2) we deduce that

$$
\widetilde{J}_{\varepsilon}(\rho)=k I(w)+\frac{1}{2}(\gamma+o(1)) e^{-\frac{\psi_{\varepsilon}(0)}{\varepsilon}}+\frac{k}{2}(\gamma+o(1)) e^{-\frac{\psi_{\varepsilon}\left(\rho e^{2 \pi \mathrm{i}}, 0\right)}{\varepsilon}}+k(\gamma+o(1)) w\left(\frac{\rho}{\varepsilon}\right),
$$

and so by (1.5) and Lemma 2.1 there exists a minimum point $\rho_{\varepsilon} \in(0,1)$. In order to get such a minimum the last two terms of $\tilde{J}_{\varepsilon}(\rho)$ have to balance, hence they must have the same order, by which, using again (1.5) and Lemma 2.1, as $\varepsilon$ goes to zero, $\rho_{\varepsilon} \rightarrow \rho^{*}$ such that

$$
2\left(1-\rho^{*}\right)=\rho^{*}
$$

and (ii) follows.

Theorem 5.3. Assume that hypotheses (f1)-(f3) hold and that $\Omega$ is the unit ball. Let $k \geq 1$ be a fixed even integer. Then, for $\varepsilon>0$ sufficiently small, the problem (1.2) has a solution $v_{\varepsilon} \in H^{2}(\Omega)$.

Furthermore there exist $P_{j}^{\varepsilon}:=\left(\rho_{\varepsilon} e^{\frac{2 \pi \mathbf{i}}{k} j}, 0\right), j=1, \ldots, k$, with $\rho_{\varepsilon} \in(0,1)$ such that, as $\varepsilon \rightarrow 0^{+}$,

(i) $v_{\varepsilon}(x)=\sum_{j=1}^{k}(-1)^{j} w\left(\frac{x-P_{j}^{\varepsilon}}{\varepsilon}\right)+o\left(e^{-\frac{\delta}{\varepsilon}}\right)$ uniformly for $x \in \bar{\Omega}$ for some $\delta>0$;

(ii) $\rho_{\varepsilon} \rightarrow \frac{2}{2+(2-2 \cos (2 \pi / k))^{1 / 2}}$ as $\varepsilon$ goes to zero.

Proof. For any even integer $k$ we look for a symmetric solution to (1.2) as in (1.10) with $P_{j}=\left(\rho e^{\frac{2 \pi \mathbf{i}}{k} j}, 0\right), \rho \in(0,1)$ and $\lambda_{j}=(-1)^{j}$ for $j=1, \ldots, k$. The rest term 
$\Phi_{\varepsilon, \mathbf{P}}$ is chosen even with respect to each $x_{h}, h=3, \ldots, N$, and, using the complex notation $z=x_{1}+\mathrm{i} x_{2}$,

$$
\Phi_{\varepsilon, \mathbf{P}}\left(z, x_{3}, \ldots, x_{N}\right)=-\Phi_{\varepsilon, \mathbf{P}}\left(z e^{\frac{2 \pi \mathrm{i}}{k}}, x_{3}, \ldots, x_{N}\right) \quad \forall\left(z, x_{2}, \ldots x_{N}\right) \in \mathbb{R}^{N} .
$$

Arguing in a standard way, we are led to find a critical point of the reduced energy

$$
\begin{aligned}
\widetilde{J}_{\varepsilon}(\rho) & =k I(w)+\frac{k}{2}(\gamma+o(1)) e^{-\frac{\psi_{\varepsilon}\left(\rho e^{2 \pi \mathbf{i}, 0}\right)}{\varepsilon}}-k(\gamma+o(1)) \sum_{j=1}^{k-1} \lambda_{j} w\left(\rho \frac{\left|e^{2 \pi \mathbf{i}}-e^{\frac{2 \pi \mathbf{i}}{k} j}\right|}{\varepsilon}\right) \\
& =k I(w)+\frac{k}{2}(\gamma+o(1)) e^{-\frac{\psi_{\varepsilon}\left(\rho e^{2 \pi \mathrm{i}}, 0\right.}{\varepsilon}}+2 k(\gamma+o(1)) w\left(\rho \frac{\left(2-2 \cos \frac{2 \pi}{k}\right)^{1 / 2}}{\varepsilon}\right),
\end{aligned}
$$

where the previous expansion holds uniformly on compact sets of $(0,1)$. Therefore by (1.5) and Lemma 2.1 there exists a minimum point $\rho_{\varepsilon}$. In order to get such a minimum the last two terms of $\tilde{J}_{\varepsilon}(\rho)$ have to balance, consequently they must have the same order, by which, again using (1.5) and Lemma 2.1, as $\varepsilon$ goes to zero, $\rho_{\varepsilon} \rightarrow \rho^{*}$ such that

$$
2\left(1-\rho^{*}\right)=\rho^{*}(2-2 \cos (2 \pi / k))^{1 / 2},
$$

and (ii) follows.

\section{ACKNOWLEDGEMENTS}

The authors are very grateful to Professor Juncheng Wei for suggesting the problem and for several useful discussions.

\section{REFERENCES}

[1] T. Bartsch, T. Weth. The effect of the domain's configuration space on the number of nodal solutions of singularly perturbed elliptic equations. Topol. Methods Nonlinear Anal. 26 (2005), 109-133. MR2179353 (2006k:35076)

[2] T. Bartsch, T. Weth. Three nodal solutions of singularly perturbed elliptic equations on domains without topology. Ann. Inst. H. Poincaré Anal. Non Linéaire 22 (2005), 259-281. MR.2136244 (2006m:35120)

[3] V. Benci, G. Cerami. Positive solutions of some nonlinear elliptic problems in exterior domains. Arch. Rational Mech. Anal. 99 (1987), 283-300. MR898712 (88f:35014)

[4] D. Cao, N. Dancer, E. Noussair, S. Yan. On the existence and profile of multi-peaked solutions to singularly perturbed semilinear Dirichlet problems. Discrete Contin. Dynam. Systems 2 (1996), 221-236. MR:1382508 (96m:35095)

[5] G. Cerami, J. Wei. Multiplicity of multiple interior peak solutions for some singularly perturbed Neumann problems. Internat. Math. Res. Notices 12 (1998), 601-626. MR 1635869 $(99 \mathrm{j}: 35063)$

[6] F.H. Clarke. Optimization and nonsmooth analysis. John Wiley and Sons, 1983. MR709590 (85m:49002)

[7] N. Dancer. The effect of domain shape on the number of positive solutions of certain nonlinear equations. I. J. Differential Equations 74 (1988), 120-156. MR.949628 (89h:35256)

[8] N. Dancer. The effect of domain shape on the number of positive solutions of certain nonlinear equations. II. J. Differential Equations 87 (1990), 316-339. MR.1072904 (91j:35034)

[9] N. Dancer, S. Yan. A singularly perturbed elliptic problem in bounded domains with nontrivial topology. Adv. Differential Equations 4 (1999), 347-368. MR.1671254 (2000d:35009)

[10] N. Dancer, S. Yan. Effect of the domain geometry on the existence of multipeak solutions for an elliptic problem. Topol. Methods Nonlinear Anal. 14 (1999), 1-38. MR.1758878 (2001b:35106)

[11] N. Dancer, J. Wei. On the effect of domain topology in a singular perturbation problem. Topol. Methods Nonlinear Anal. 11 (1998), 227-248. MR1659466 (2000a:35012) 
[12] T. D'Aprile, A. Pistoia. Existence, multiplicity and profile of sign-changing clustered solutions of a semiclassical nonlinear Schrödinger equation. Ann. Inst. H. Poincaré, Anal. Non Linéaire 26 (2009), 1423-1451. MR.2542732 (2010j:35178)

[13] T. D'Aprile, A. Pistoia. Nodal clustered solutions for some singularly perturbed Neumann problems, Comm. Partial Differential Equations 35 (2010), 1355-1401.

[14] M. del Pino, P. Felmer, J. Wei. Multi-peak solutions for some singular perturbation problems. Calc. Var. Partial Differential Equations 10 (2000), 119-134. MR.1750734 (2001a:35019)

[15] M. del Pino, P. Felmer, J. Wei. On the role of distance function in some singular perturbation problems. Comm. Partial Differential Equations 25 (2000), 155-177. MR 1737546 (2000m:35017)

[16] B. Gidas, W. M. Ni, L. Nirenberg. Symmetry of positive solutions of nonlinear elliptic equations in $\mathbb{R}^{N}$. Adv. Math. Suppl Stud. 7A (1981), 369-402. MR634248 (84a:35083)

[17] M. Grossi, A. Pistoia. On the effect of critical points of distance function in superlinear elliptic problems. Adv. Differential Equations 5 (2000), 1397-1420. MR.1785679 (2001j:35111)

[18] M. Grossi, A. Pistoia, J. Wei. Existence of multipeak solutions for a semilinear Neumann problem via nonsmooth critical point theory. Calc. Var. Partial Differential Equations 11 (2000), 143-175. MR1782991 (2001h:35012)

[19] C. Gui, J. Wei. Multiple interior peak solutions for some singularly perturbed Neumann problems. J. Differential Equations 158 (1999), 1-27. MR.1721719 (2000g:35035)

[20] C. Gui, J. Wei. On multiple mixed interior and boundary peak solutions for some singularly perturbed Neumann problems. Canad. J. Math. 52 (2000), 522-538. MR1758231 (2001b:35023)

[21] Y. Li, L. Nirenberg. The Dirichlet problem for singularly perturbed elliptic equations. Comm. Pure Appl. Math. 51 (1998), 1445-1490. MR1639159 (99g:35014)

[22] F.H. Lin, W.M. Ni, J. Wei. On the number of interior peak solutions for a singularly perturbed Neumann problem. Comm. Pure Appl. Math. 60 (2007), 252-281. MR.2275329(2008k:35161)

[23] W.M. Ni, J. Wei. On the location and profile of spike-layer solutions to singularly perturbed semilinear Dirichlet problems. Comm. Pure Appl. Math. 47 (1995), 731-768. MR.1342381 (96g:35077)

[24] E. Noussair, J. Wei. On the effect of domain geometry on the existence and profile of nodal solutions of some singularly perturbed semilinear Dirichlet problem. Indiana Univ. Math. J. 46 (1997), 1255-1272. MR.1631584 (99e:35011)

[25] J. Wei. Conditions for two-peaked solutions of singularly perturbed elliptic equations. Manuscripta Math. 96 (1998), 113-136. MR.1624364 (99e:35014)

[26] J. Wei. On the interior spike solutions for some singular perturbation problems. Proc. Roy. Soc. Edinburgh Sect. A 128 (1998), 849-874. MR1635448 (99h:35012)

[27] J. Wei, M. Winter. Symmetry of nodal solutions for singularly perturbed elliptic problems on a ball. Indiana Univ. Math. J. 54 (2005), 707-741. MR2151231(2006b:35132)

Dipartimento di Matematica, Università di Roma "Tor Vergata", via della Ricerca SCIEntifica 1, 00133 Roma, Italy

E-mail address: daprile@mat.uniroma2.it

Dipartimento di Metodi e Modelli Matematici, Università di Roma "La Sapienza", via Antonio Scarpa 16, 00161 Roma, Italy

E-mail address: pistoia@dmmm.uniroma1.it 